\title{
AUTONOMOUS REFRIGERATOR ROBOT WITH PICK AND PLACE OPERATION
}

\author{
Aditya. $\mathbf{V}^{1 *}$, Rizwan.S $\mathbf{S}^{2}$ \\ *1,2Student, Department of Mechatronics Engineering Mahatma Gandhi Institute of Technology, Hyderabad, India \\ *1Email: vaditya62@gmail.com, ${ }^{2}$ Email: ezdu50@gmail.com
}

*Corresponding Author: -

Email:vaditya62@gmail.com

\begin{abstract}
:
One of the most common pieces of equipment found in all biomedical and research laboratories are the specimen refrigerator. While seeming not as glamorous or fancy as some of the other laboratory equipment, the specimen refrigerator plays a crucial role in laboratory research. Almost all experiment involving living cultures or specimens require them to be maintained at a specific temperature. Sometimes the temperature change can be for preservation purposes and sometimes it might be to simulate an actual environmental condition. Nevertheless, temperature control of experiments is of outmost importance in any modern research facility.

When working with specimens in a laboratory it is often necessary to process samples at specific temperatures and times. In a busy biomedical lab, managing and handling all of the samples can be an overwhelming and daunting task. Different materials with varying densities can have different heat transfer coefficients as well as specific heats. With each sample put into the fridge at a different time there is almost no way to know when each sample will reach its ideal temperature. Some samples might be required to be chilled to near freezing while some might just need their temperatures reduced by a few degrees. Thus, there is a need for the device that could both measure the temperature of the samples, as well as retrieve them from the refrigerator.

The goal of our Mechatronics final project is to design and create a device capable of managing a biomedical laboratory's refrigerated sample inventory. In order to achieve this, the device needed to be able to completely operate on its own without needing the operator's input or commanding. The device would operate autonomously within an enclosed refrigerator and monitor the samples and vials being studied in the lab. When called upon to, the device would be able to retrieve a required sample based either on the time of day, time the specimen was required to be in the refrigerator or even have it removed at a set temperature point.
\end{abstract}

Key Words: Refrigerator, Robot, Bio-Medical Laboratory, Sample Specimen, Micro Controller, Density

\section{(ㄷ) (오 (1)}




\section{INTRODUCTION \\ 1.1 PURPOSE OF DEVELOPMENT}

The purpose of our project is to design and create a device capable of managing a biomedical laboratory's refrigerated sample inventory. In order to achieve this, the device needed to be able to completely operate on its own without needing the operator's input or commanding. The device would operate autonomously within an enclosed refrigerator and monitor the samples and vials being studied in the lab. When called upon to, the device would be able to retrieve a required sample based either on the time of day, time the specimen was required to be in the refrigerator or even have it removed at a set temperature point. .

\subsection{PROJECT OVER VIEW}

A mechatronics system has been developed as an alternative to the existing refrigerator and robot and this is the integration of both refrigerator as well as robot. This is designed in order to improve testing conditions in bio medical laboratories and this is developed, with the use of advanced sensors and actuators to function as a single unit.

It is completely autonomous and has numerous sensors to accommodate even the trickiest temperatures.

It is an inconvenience to pick up a test tube with exact temperature. Our robot could solve your problem. By just setting up the temperature the robotic arm picks and places the test tube required to serve the purpose.

\section{BACKGROUND}

In general, a refrigerator is a device for keeping storage items such as food, beverage, and the like, in storage for a long period of time, and refrigerating or freezing storage items according to their types desired to be kept in storage.

The refrigerator operates according to driving of a compressor provided therein. Cooling air provided to the interior of the refrigerator is generated according to a heat exchange operation of a refrigerant and continuously provided to the interior of the refrigerator according to a repeated cycling operation of compression-condensation-expansion evaporation. The provided refrigerant is evenly transferred to the interior of the refrigerator according to a convection current to allow the food items within the refrigerator to be kept at a desired temperature.

Recently, consumers demand for refrigerators that may provide a great utilization efficiency of the storage space capable of storing various storage items that change according to the elevation of the standard of living, as well as the conventional cooling efficiency, is increasing. Also, demand for various additional functions improving user convenience is increasing. For example, users are becoming interested in a robot refrigerator including a robot technique. Namely, users who want to use a refrigerator show an interest in the technique of moving and using a refrigerator by using a remote controller or a robotic arm.

\subsection{EMBEDDED SYSTEMS}

Embedded system is a combination of software and hardware designed and programmed to perform one/more particular task(s). The hardware is designed for specific application and then software is embedded in this hardware to perform the task. Both software and hardware are dedicated to that particular application. The heart of the system is either processor or controller. Processor / controller may be general purpose or special purpose that controls whole system.

There may be more than one processor/controller if system is complex. It may be possible that there is one general purpose processor / controller and one or more special purpose processors / controllers. For example, in 3G (or 4G) cell phones there is one general purpose processor that handles user commands, memory and display etc. And there are special purpose processors like DSP for voice communication and network management, display controller to generate real and reach images on color LCD screen.

An embedded system is a special-purpose system in which the computer is completely encapsulated by or dedicated to the device or system it controls. Physically embedded systems range from portable devices such as digital watches and MP3 players, to large stationary installations like traffic lights, factory controllers, or the systems controlling nuclear power plants. In terms of complexity embedded systems can range from very simple with a single microcontroller chip, to very complex with multiple units, peripherals and networks mounted inside a large chassis or enclosure.

\subsection{NEED OF THE PROJECT}

The most complex, and therefore most marketable, use for our device would be in a scenario where a variety of samples and cultures are being studied simultaneously. In a scenario such as this one, where different specimens are required to be controlled at similar temperatures, figuring out exactly how long and at what time each sample will reach its set temperature can be a task that can take a dedicated researcher all day to achieve. By incorporating our device into the lab, the researchers are free to take care of more pressing devices while our machine monitors all of the specimens. Without any effort at all it can not only monitor each of the specimens' temperatures, but remove them from the refrigerator when the desired temperature is reached and place it on the lab table, alerting the researcher that his specimen is ready. This type of control and operation can allow optimal efficiency and productivity in the lab.

\subsection{REFRIGERATOR OF THE FUTURE}

While the Refrigerator Robot is currently only a concept, with more and more advances in technology and a greater need for smaller appliances, the Refrigerator Robot may very well be available for use in the near future. With its unique size, shape, and way of storing specimens and use of the robotic arm by detecting the exact test tube with set temperature the Refrigerator Robot is definitely one of the most unusual inventions of its time. 


\section{DESIGN AND CONSTRUCTION}

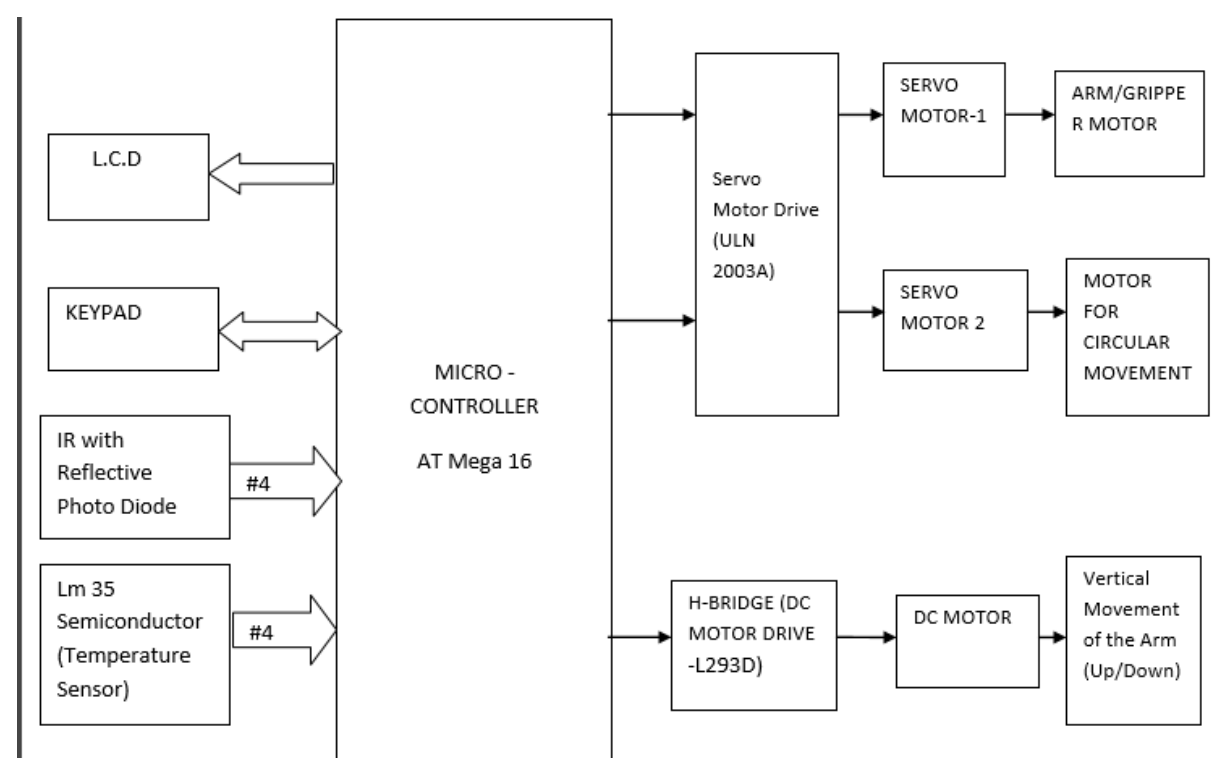

Figure 1: Functional Block Diagram of the Project

The block diagram represents the autonomous refrigerator robot. The input to the system is temperature that we give on the keypad. There are various sensors such as photo resistors and thermistors. Photo Resistors are used to check whether the slot is filled or not. In order for the robot to understand when and where a new specimen was placed into the refrigerator, a sensor system needed to be set up. The system allows the robot to identify in what locations and when an object was placed in a given location with the use of a photo resistor. A photo resistor is a resistor that can change its resistance based on a light incident on its surface. A photo resistor placed in a dark environment will have a very large resistance, possibly in the Mega ohm range, while the same resistor placed in a well-lit area can have a resistance as small as one K-ohm. LM 35 temperature sensor is placed in order to sense the temperature of the specimen. The micro controller reads the input given and works on the program specified earlier and depending upon the condition satisfied, it works on the statement specified within the condition. The output signal is sent to the servo motor as well as dc motor. The signal given to servo motor is used to drive the gripper/arm and circular movement and also for circular movement. The signal given to dc motor is used for vertical movement of the arm and it indeed drives the $\mathrm{H}$ bridge where it is used to control the speed and movement of the arm in vertical directions.

\subsection{STEPS OF FABRICATION}

- Presto Teak box is taken and is cut according to the required dimensions.

- Holes are drilled in a semi-circular arc for placing the specimens (test tubes).

- Servo Motor is mounted on an acrylic sheet.

- Dc Motor is mounted using various acrylic sheets and mild steel rods.

- Servo Motor is placed inside the presto teak box along with dc motor casing.

- Robotic Arm along with another servo in the proper position is placed in the dc motor casing and fastened tightly with nuts and bolts.

- The micro controller is placed in between the slot present in the compressed wood and all the connections are given to it accordingly.

- Another teak of PVC material is taken and it is cut accordingly to accommodate LCD and key pad.

- All the connections are given accurately.

\subsection{OPERATION}

The robotic arm is developed to work in cold, closed environments. For its proper operation, the surroundings must be clean and free of any kind of obstacles that could stop it from moving on the trails, or hitting objects while moving the arm. During the execution of a function, the robot must not be touched or pushed in any ways. Unexpected contact may cause permanent malfunction.

\section{Selecting a Function}

To start the operation, the robot must be turned on. This is done by changing the position of the switch from 0 to 1 . The LCD display will then list the functions. The user must wait until the message "Select Function" is shown to start pressing the buttons. Button 1 is used to select the function, where pressing the button increases the number until the user reaches the desired number. Button 2 enters the selection and starts operation.

\section{Placing the Test Tubes}

To make sure the arm will detect, sense, and lift the test tubes properly, every can must be placed carefully on the 
determined spot. There are 3 possible positions, each one with its own circular pad. The user must place the test tube in the space (supplied) and, then, position it adequately on the pad. The arm will automatically detect the positions and perform the selected functions. The arm makes possible for the robot to lift the can, being an important part of the product.

\section{RESULTS AND DISCUSSION}

\subsection{RESULTS}

The autonomous refrigerator robot system is constructed and is tested in different modes.

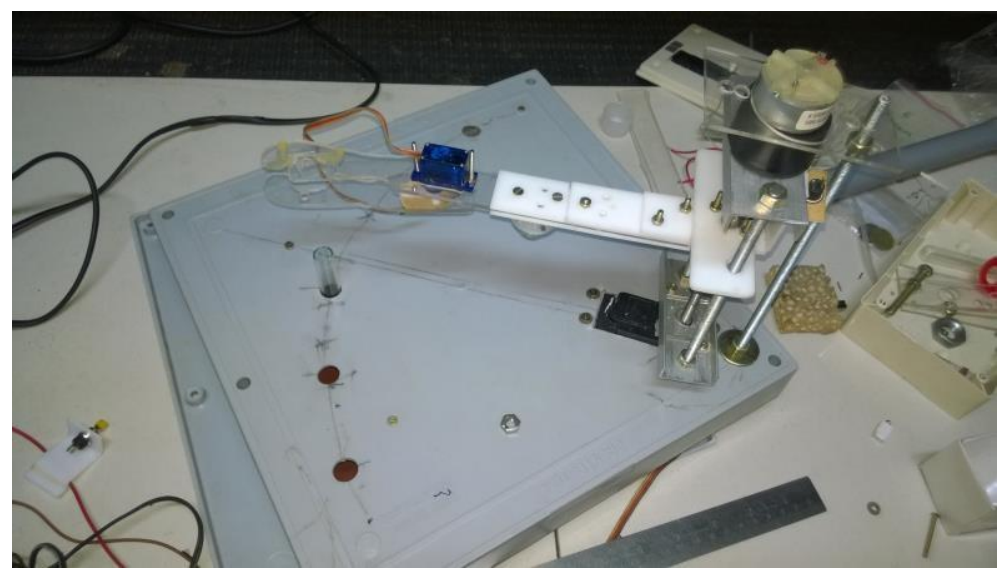

Figure 2: Completed Mechanical Structure

The final robotic arm is tested over various temperatures of specimens and its operation is found to be satisfactory. As soon as we set the mode whether manual or automatic the robotic arm goes to its home position and picks up the test tube according to the set temperature.

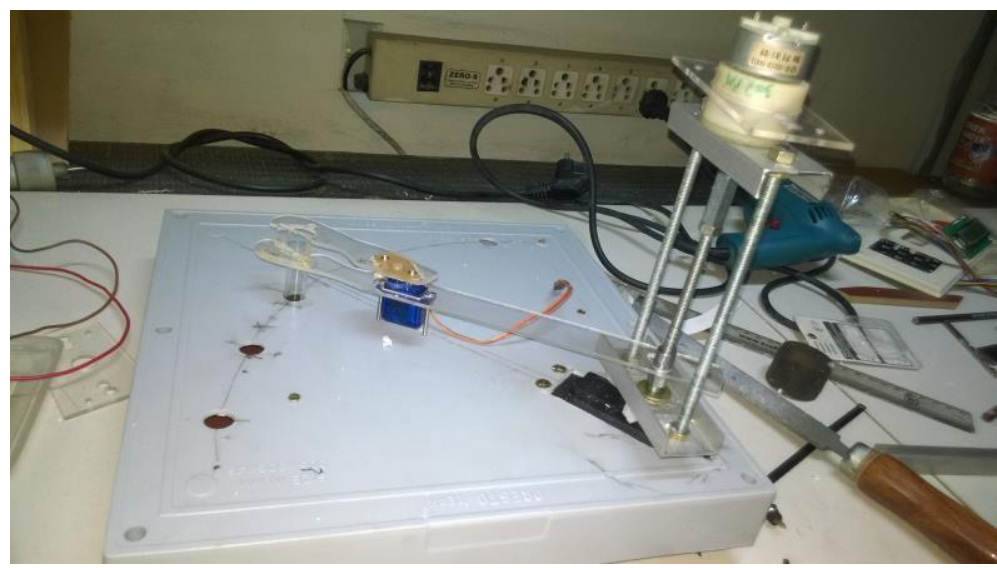

Figure 3: Lifting the Test tube

The robotic arm with the help of servo motor grips the test tube and dc motor aids in lifting it up and another servo motor rotates at an angle in such a way that it places the test tube at the proper place. 


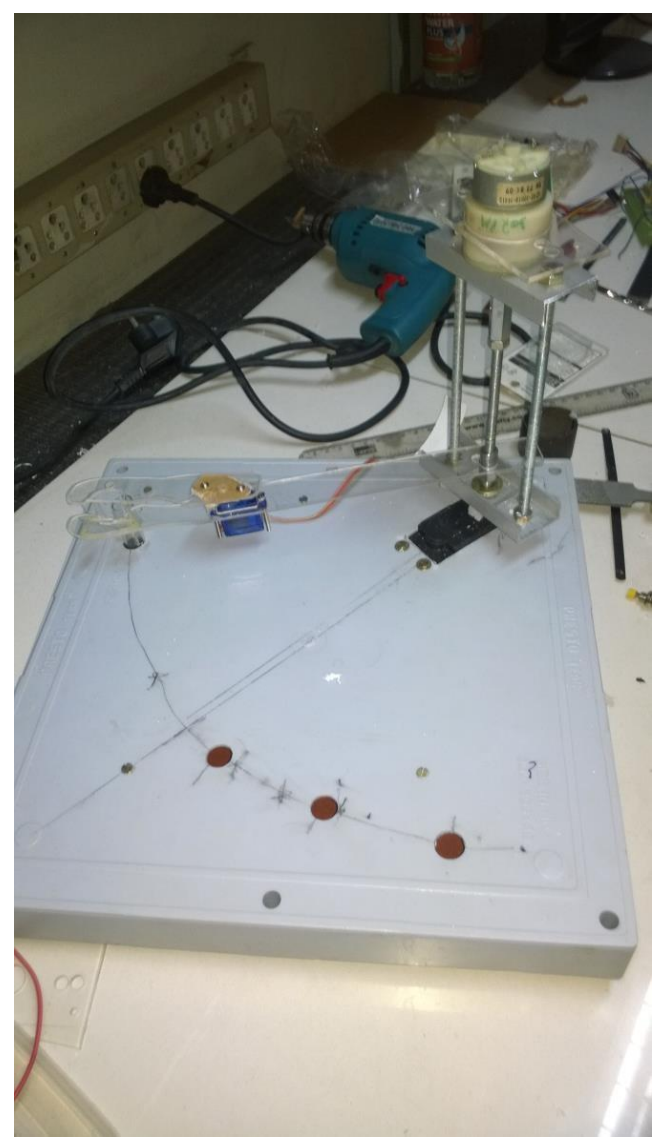

Figure 4: Placing the Test tube

After execution of the task the robotic arm goes back to the home position.

\subsection{GANTT CHART}

A Gantt chart is a type of bar chart, adapted by Karol Adamiecki in 1896 and independently by Henry Gantt in the 1910s, that illustrates a project schedule. Gantt charts illustrate the start and finish dates of the terminal elements and summary elements of a project. Terminal elements and summary elements comprise the work breakdown structure of the project. Modern Gantt charts also show the dependency (i.e., precedence network) relationships between activities.

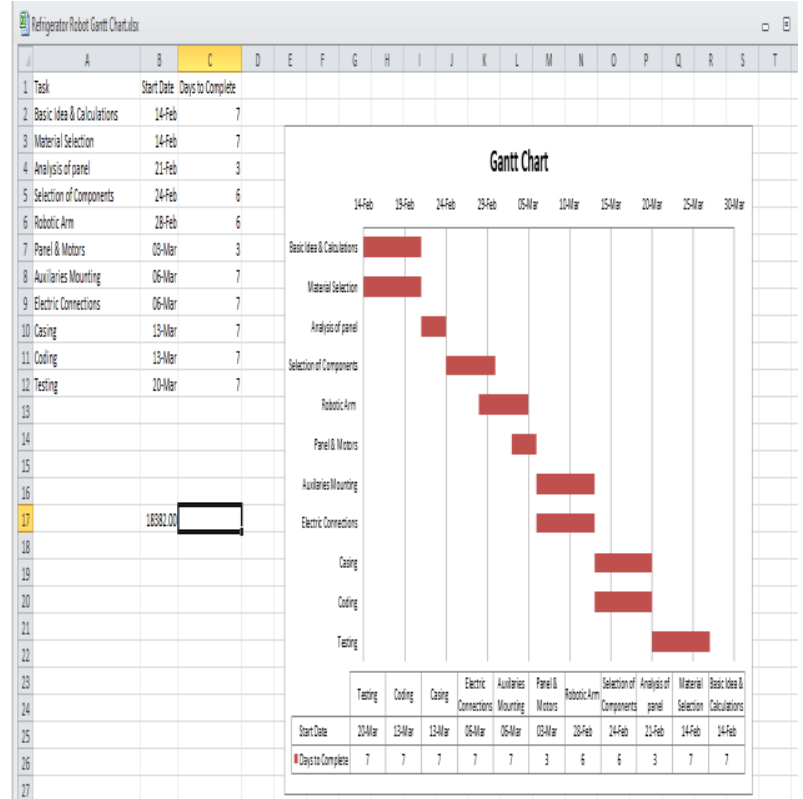

Figure 5: Gantt Chart

The Gantt chart is all about how we divided the work against how many days. Right from idea to testing of prototype it took almost 40 days to complete it. 


\section{DEVELOPMENTS}

Although prototyped as a "refrigerator insert", the automatic refrigerator device can be built directly into a larger more spacious laboratory refrigerator. When not being used as an insert, the device can be incorporated into numerous shelves and spaces. This will allow to device to monitor dozens, if not hundreds, of specimens and cultures. One shortcoming of our device was our limited access to microcontroller devices. The basic stamp is built with only a limited number of input and output pins available. This shortcoming severely limits our device's ability to monitor large number of samples.

Another manufacturing hurdle that would need to be overcome for a retail prototype would be materials. Our robot was build almost entirely out of mild steel and acrylic sheet. While using acrylic sheet allowed us to build an operational and fully integrated robot in a short amount of time, it is limited in its compactness and weight. A robot made entirely of aluminium would be able to be built stronger, lighter and smaller. The prototype we designed would require a large refrigerator for only a few samples, while a smaller more compact device would take up a smaller footprint within the refrigerator allowing for more capability. A properly designed and constructed device wouldn't take up much more space than the samples it is programmed to monitor.

In addition, the temperature of the samples we were demonstrating was taken using a thermistor. While not a rare electrical component, it is not considered to be a mainstream electrical device. This severely limited our abilities to find a device with the response time and range our device fully required. We found our thermistor to have a steady state response time of approximately 20 seconds. While adequate for the prototype, in a retail model a quicker and more accurate thermistor could be used, perhaps one requiring only a few seconds to reach steady state conditions.

\section{APPLICATIONS}

- A variety of samples can be studied simultaneously.

- It is mainly used in Bio-Medical Laboratories, Industries, Universities, Research Institutions.

- Without any effort at all it can not only monitor each of the specimens' temperatures, but removes them from the refrigerator when the desired temperature is reached and places it on the lab table, alerting the researcher that his specimen is ready.

- The flexibility of incorporating a micro controller into the device allows it to be configured to various users' preferences.

\section{IMPROVEMENTS}

Even though the emergency button allows the user to stop the execution of the program if a problem occurs, an accidental reset due to random issues would make the robot lose track of its position. The addition of an arm position, a claw state and track position sensors would solve this issue, making possible an initialization routine that would take the robot back to a standard initial state.

Additionally, a better organization of the cables, maybe using a bigger breadboard, would make the prototype a lot better looking, easier to handle, easier to maintain and would avoid problems like wires unintentionally short-circuited.

\section{CONCLUSION}

The refrigerator robot can prove to be an asset to any biomedical or university lab throughout the country. The device can allow researchers to focus on important tasks while taking care of ensuring specimens and cultures are properly taken care of. In addition to increasing productivity and performance, the device can help ensure that laboratory errors are left frequent and more controlled. Often a researcher will forget about a sample in a refrigerator or not store it in the precise temperature required. The automatic refrigerator device will remove the various errors associated with precious specimen temperature control. Once properly constructed and manufactured, the automatic specimen refrigerator device is a machine that no laboratory will be found without.

\section{REFERENCES}

[1].C. C. Consortium, “A Roadmap for US Robotics: From Internet to Robotics,” Tech. Rep., May 212009.

[2].E. R. T. Platform, "Robotic Visions To 2020 And Beyond - The Strategic Research Agenda for Robotics in Europe," Tech. Rep., June 2009.

[3].U. Hillenbrand, B. Brunner, C. Borst, and G. Hirzinger, "The Robutler: a Vision-Controlled Hand- Arm System for Manipulating Bottles and Glasses," in 35th International Symposium on Robotics, 2004.

[4].R. Lienhart and J. Maydt, "An extended set of haar-like features for rapid object detection," in ICIP, 2002. 